\title{
FORUM
}

Reçu le 27.08.2013. Approuvé le 09.01.2014

Accepté dans le cadre d'une procédure d'évaluation en double aveugle. Éditeurs Scientifiques: Ernesto R. Gantman, Helà Yousfi et Rafael Alcadipani

DOI: http://dx.doi.org/10.1590/So034-759020150206

\section{L'APPORT DE LA SOCIOLOGIE PRAGMATIQUE FRANÇAISE AUXÉTUDES CRITIQUES EN MANAGEMENT}

\author{
Contribuição da sociologia pragmática francesa para os estudos críticos em \\ Administração \\ The contribution of French pragmatic sociology to critical Management studies
}

\section{RÉSUMÉ}

La sociologie pragmatique française (SP), inspirée des travaux de Luc Boltanski et Laurent Thévenot, est de plus en plus utilisée par les études organisationnelles en management. Pourtant, la dimension critique de cette approche n'a pas encore été intégrée au profit de la connaissance en management et organisations (MOK). Dans cet article, nous explicitons l'apport que cette sociologie peut représenter pour les études critiques en management (CMS). En tant que science de la science des acteurs, nous suggérons que la SP est fertile pour développer une approche de performativité critique. En particulier, nous démontrons que l'approche permet de mettre en lumière les nouvelles formes de domination plus complexes s'exerçant dans les organisations contemporaines. En utilisant des études empiriques d'organisations mobilisant la SP, nous montrons comment les concepts de compromis et d'épreuve développés par cette approche présentent un outillage permettant de renouveler la critique des organisations au profit de la MOK.

MOTS CLÉS | Sociologie pragmatique française, Sociologie de la critique, performativité critique, études organisationnelles en management, études critiques en management.

\section{RESUMO}

A sociologia pragmática francesa (SP), inspirada nos trabalhos de Luc Boltanski e Laurent Thévenot, é cada vez mais utilizada pelos estudos organizacionais em Administração. No entanto, a dimensão crítica dessa abordagem ainda não foi integrada para o proveito do conhecimento em Administração e organizações (MOK). Nesse artigo, esclarecemos a contribuição que essa sociologia pode apresentar para os estudos críticos em Administração (CMS). Enquanto ciência da ciência dos atores, sugerimos que a "SP" é fecunda por desenvolver uma abordagem de performatividade crítica. Particularmente, demonstramos que tal abordagem permite trazer à luz as novas formas de dominação mais complexas exercidas nas organizações contemporâneas. Através de estudos empíricos de organizações que mobilizam a "SP", mostramos como os conceitos de compromisso e de prova desenvolvidos por essa abordagem apresentam ferramentas permanentes para se renovar a crítica das organizações em benefício da "MOK".

PALAVRAS-CHAVE / Sociologia pragmática francesa, Sociologia da crítica, performatividade crítica, estudos organizacionais em administração, estudos críticos em Administração.

\begin{abstract}
French pragmatic sociology (PS), inspired by the work of Luc Boltanski and Laurent Thévenot, is being increasingly used by organizational management studies. However, the critical dimension of this approach has not yet been integrated for the benefit of knowledge in management and organizations (MOK). In this article, we elaborate on the contribution that PS can represent for critical management studies (CMS). As a 'science of the players' science' [science de la science des acteurs], we suggest that PS is fertile for the development of the concept of critical performativity. In particular, we demonstrate that the approach allows shedding light on the new and more complex forms of domination exerted in contemporary organizations. Using empirical studies of organizations employing PS, we demonstrate how the concepts of compromise and test developed by this approach offer tools that allow renewing the critique of organizations for the benefit of MOK.
\end{abstract}

KEYWORDS / French pragmatic sociology, sociology of critique, critical performativity, organizational management studies, critical management studies.

BENJAMIN TAUPIN

benjamin.taupin@cnam.fr

Maître de conférences au Conservatoire national des arts et métiers - Paris, France 


\section{INTRODUCTION}

Prenant son origine dans les Économies de la Grandeur (Boltanski \& Thévenot, 1991), le courant de la SP est une approche sociologique qui gagne en reconnaissance dans le domaine des sciences humaines et sociales. Également appelée sociologie de la critique, la SP se donne comme « objet préférentiel la description et l'analyse de la manière dont les acteurs "eux-mêmes" désignent les êtres qui composent leur environnement (...) et, ce faisant, contribuent à performer le monde social " (Boltanski, 2012, pp. 340-341). La méthode de cette sociologie consiste à étudier les discours des acteurs élaborés dans leur recherche d'un bien commun, c'est-à-dire dans la poursuite de ce qu'ils considèrent comme juste. Elle permet de mettre l'accent sur la créativité et sur l'inventivité des acteurs et de rendre sensible l'intelligence du social dont ils font preuve - quels qu'ils soient (Blokker, 2011).

Dans cet essai, nous explorons le potentiel de la SP en tant que théorie critique des organisations. Nous mettons en exergue la capacité de ce cadre à analyser de nouvelles formes de domination organisationnelle. En effet, l'utilisation de la sociologie de la critique de Boltanski et Thévenot met à disposition des outils concrets pour le chercheur voulant développer une approche critique en sciences de gestion. Dans ce contexte, l'emploi des notions de compromis et d'épreuve dans une démarche générale faisant l'hypothèse de relative liberté des acteurs permet la mise en avant de processus de domination complexe.

Nous recourrons au cadre d'analyse du caractère critique des recherches en MOK de Fournier et Grey (2000) et la notion de performativité critique (Spicer, Alvesson, \& Kärreman, 2009 ; Alvesson \& Spicer, 2012) avec ses prolongements (Alcadipani \& Hassard, 2010) pour circonscrire les caractéristiques des CMS. Une fois les éléments constitutifs du caractère critique des analyses en management définis, nous montrerons que l'approche de la SP dispose de ces éléments et peut par conséquent représenter un axe de développement prometteur au profit des études critiques en management.

\section{ÉPREUVES, COMPROMIS ET QUALIFICATION DANS LES ORGANISATIONS}

Les études organisationnelles ont été influencées par la SP. Une revue de la littérature empirique organisationnelle inspirée par les Économies de la Grandeur de Boltanski et Thévenot a été réalisée par Jagd (2011). Ce dernier souligne la référence de McInerney aux Cités dans son travail ethnographique (McInerney, 2008) ainsi que le lien historique entre Stark et la SP (Stark, 2000 ; Stark, 2009). Jagd mentionne également le recours à ce cadre par le groupe de travail de Joanne Roch (Roch, 2005 ; Boivin \& Roch, 2006) sur les relations inter-organisations et les fusions-acquisitions. Enfin il utilise l'approche de Boltanski et Thévenot afin d'expliquer la résistance aux stratégies de changement dans les grandes entreprises françaises (Fronda \& Moriceau, 2008). Depuis les premières références du potentiel de cette approche (Biggart \& Beamish, 2003 ; Denis, Langley, \& Rouleau, 2007 ; Thornton \& Ocasio, 2008), l'intérêt de la communauté des études organisationnelles pour celle-ci a entraîné une production croissante de travaux utilisant la SP, pour la compréhension de phénomènes dans des domaines aussi divers que la comptabilité (Annisette \& Richardson, 2011), les ressources humaines (Pernkopf-Konhaeuser \& Brandl, 2010), la création d’un marché pour les dérivés climatiques (Huault \& Rainelli-Weiss, 2011). Cet engouement s'est aussi traduit par l'émergence de travaux utilisant ce cadre de manière centrale pour l'analyse des organisations. Patriotta, Gond, et Schultz (2011) se sont fondés sur les justifications sur lesquelles les acteurs s'appuient pour réaffirmer la légitimité en vigueur dans le domaine de l'énergie nucléaire. Les auteurs ont ainsi pu mettre à profit la théorie de la Justification afin d'instruire la problématique du maintien institutionnel. Le maintien apparaît alors comme un processus soutenu par des controverses et des justifications publiques au cours desquelles les acteurs font appel à leur sens moral. La proximité des approches entre néo institutionnalisme et Économies de la Grandeur a mené à tenter de rapprocher le florissant essor de la notion de logique institutionnelle de cette SP (Cloutier \& Langley, 2013). Une édition spéciale sur le néo-institutionnalisme a confirmé l'attrait de l'étude des organisations en articulant néo-institutionnalisme et Économies de la Grandeur (Dansou \& Langley, 2012 ; Taupin, 2012). Cependant, si l'on excepte un travail de déconstruction de l'établissement des prix dans le commerce équitable (Reinecke, 2010), les études organisationnelles n'ont pas saisi l'approche issue des Économies de la Grandeur dans leur dimension critique. Plusieurs voix ont ainsi récemment appelé (Dunne, 2012 ; Cloutier \& Langley, 2013, p. 17) au développement d'un agenda de recherche visant au rapprochement entre la SP et les l'analyse des mécanismes d'oppression au profit des études organisationnelles.

Pour la SP, le fait pour les acteurs de recourir à des principes moraux pour justifier leurs propos est exprimé par la notion de remontée en généralité. Dans leur ouvrage séminal, Boltanski et Thévenot construisent six mondes qui rendent compte des registres d'argumentation utilisés par les acteurs 
lorsqu'ils remontent en généralité : le monde de l'inspiration, le monde domestique, le monde de l'opinion (ou du renom), le monde civique, le monde industriel et le monde marchand. L'ouvrage de Boltanski et Chiapello (1999) détermine une cité supplémentaire : le monde par projet. Claudette Lafaye et Laurent Thévenot (1994) ont quant à eux évoqué le monde de la cité verte. Dans leur démarche de justification lors des situations de dispute, les acteurs mobilisent les objets, matériels ou symboliques, en fonction des principes supérieurs auxquels ils font appel. La construction de leur argumentation va ainsi correspondre à des mondes (Boltanski \& Thévenot, 1991). Ainsi, en rapport à un principe de généralité spécifique, l'appartenance à un monde détermine ce que l'on nomme la qualification des objets et des personnes ainsi que leurs relations (voir la figure 1 ci-dessous). Par exemple, ce qui est grand dans le monde industriel, c'est le caractère performant et efficace. À l'inverse, quelqu'un d'inefficace sera petit dans ce monde. Dans le monde inspiré, la grandeur dépendra de la capacité à être créatif et inspiré et le fait d'avoir « les pieds sur terre » correspondra à une forme de dégénérescence. Dans le cadre tracé par Boltanski et Thévenot, la grandeur est « la façon dont on exprime les autres, dont on les incarne, dont on les comprend ou encore dont on les représente (autant de modalités qui dépendent de la cité considérée) » (Boltanski \& Thévenot, 1991, p. 167). La justice apparaît n'exister dès lors que dans une relation située, contextualisée et spécifique.

Figure 1. Les six mondes des Économies de la Grandeur

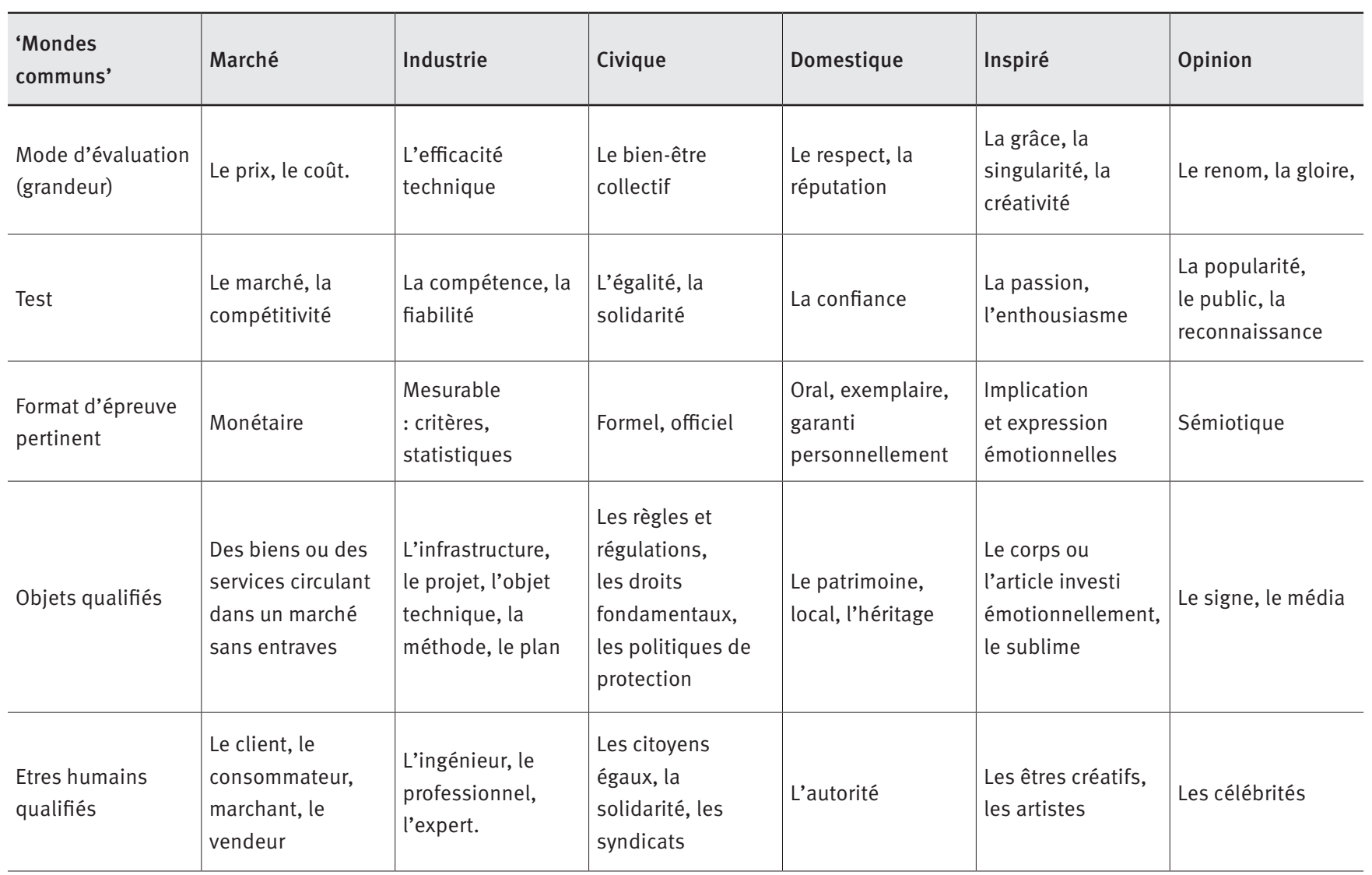

Source : Adapté de Thévenot, Moody et Lafaye (2000, p. 241). Traduction de l'auteur.

Un malentendu courant à propos de l'utilisation de la SP consiste à l'utiliser comme un outil de classification des justifications (Breviglieri, Lafaye, \& Trom, 2009). Or elle représente davantage qu'un simple outil de classification des discours : elle étudie la dynamique de l'accord, le processus qui engage l'ajustement des acteurs dans la vie sociale et mène à la constitution de mondes communs relativement stables. Au-delà du répertoire des cités, dont disposent les acteurs et qui semble donné, l'intérêt de cette sociologie provient de la mise en lumière de ce processus à travers les notions fondamentales d'épreuve et de compromis (Nachi, 2006 ; Breviglieri, Lafaye, \& Trom, 2009 ; Dansou \& Langley, 2012). En mobilisant ces concepts, Taupin (2012) a résumé le processus de qualification que nous venons de décrire en explicitant les différents niveaux organisationnels et les éléments institutionnalisés (voir la figure 2 ci-dessous). 
Figure 2. La circulation de la critique à travers les niveaux organisationnels

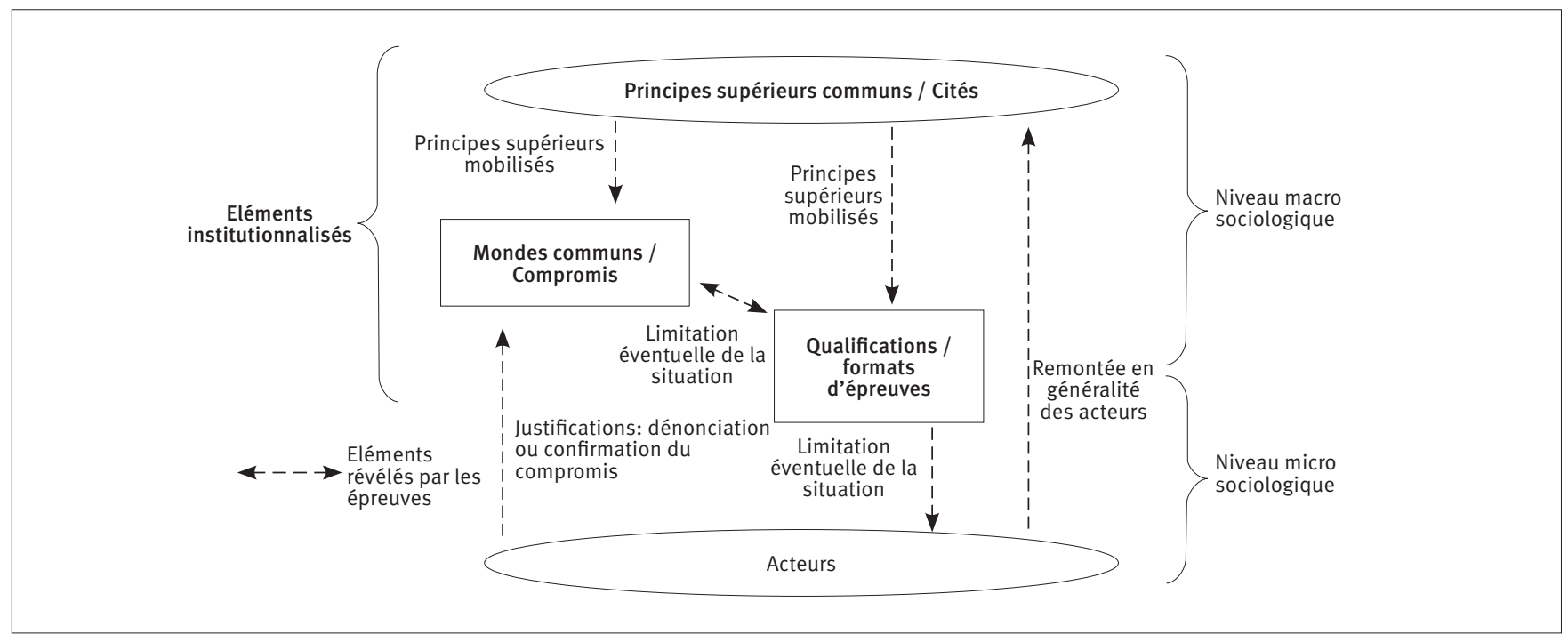

Source : Taupin (2012)

La notion de compromis constitue la pierre angulaire des Economies de la Grandeur (Nachi, 2004 ; Nachi, 2006). A travers la figure du compromis, il s'agit pour les individus de réduire les tensions entre des mondes multiples et d'en extraire plusieurs principes d'équivalence. Le compromis permet ainsi la coexistence de différents intérêts et manières de penser en mettant en place un vivre ensemble au-delà des oppositions indépassables. Boltanski et Thévenot (1991, p. 175) écrivent en ce sens : «La multiplication des objets composites qui se corroborent et leur identification à une forme commune contribuent ainsi à stabiliser, à frayer le compromis. Lorsqu'un compromis est frayé, les êtres qu'ils rapprochent deviennent difficilement détachables ». Afin que le bonheur des grands puisse profiter au bonheur des petits, le compromis doit permettre de faire coexister différents mondes, accroissant l'état de justice pour les acteurs. Il est par conséquent directement associé au principe du bien commun dans les Économies de la Grandeur. De ce caractère composite, le compromis tire une fragilité qui ne le rend pas pour autant éphémère. Comme l'indique la citation ci-dessus, le compromis peut être renforcé et donc apparaître comme non contesté. Lorsque le bien commun est atteint, la recherche des intérêts individuels est dépassée dans un compromis reconnu publiquement qui permet de sortir des disputes. Néanmoins, comme le rappelle Ricœur (1991, p. 3), « le compromis est toujours faible et révocable, mais c'est le seul moyen de viser le bien commun ». Le compromis peut donc être ébranlé à tout moment en étant dénoncé. Par conséquent, dans le cadre de la SP, le terme de solidité ne peut que difficilement convenir pour décrire le compromis. Il s'agit d'une apparence de stabilité dans laquelle demeure la forme dynamique incarnée par l'opposition ontologique entre les grandeurs irréconciliables associées dans un compromis.

L'exemple des établissements de crédit implantés dans des zones rurales permet d'illustrer cette notion. Des dispositifs composites sont mis en place (Wissler, 1989) pour permettre des points de passages entres les logiques relevant du monde domestique (l'ancrage local incarné par les relations amicales, familiales, de voisinage, de services) et celles relevant du monde industriel (volonté d'objectiver la décision d'octroi de crédit). Ainsi la décision de crédit finale dépasse la situation inextricable pour trouver un accord plus général qui réduit les tensions antérieures (Wissler, 1989, p. 113. La lettre en exposant indique la première lettre du monde de référence, I pour monde industriel, D pour monde domestique) :

l'analyste financier construit un plan' de consolidation financière ${ }^{\mathrm{D}-\mathrm{I}}$ à moyen termel. II demande, en faisant valoir l'autorité ${ }^{\mathrm{D}}$ du bailleur de fonds, à la famille ${ }^{D}$ de s'engager davantage dans l'entreprise par une dotation en capital et un apport sur compte bloqué, pour prévenir le risque de détérioration des comptes' (...).

L'étude du processus d'octroi de crédit montre comment l'établissement bancaire a dû faire un compromis entre deux logiques d'action dans son fonctionnement. L'accord permet de dépasser la situation d'opposition entre l'analyse financière, relevant du monde industriel, et les recommandations du conseil d'administration local, engagé jusque-là dans une logique de jugement domestique. La nouvelle équivalence plus générale 
établie ici s'incarne dans de nouveaux objets composites qui renforcent le compromis : on demandera en contrepartie du crédit une caution'-solidaire ${ }^{\mathrm{D}}$ consolidant l'accord réalisé entre monde industriel et monde domestique.

Pour la SP, le concept fondamental d'épreuve permet d'insister sur l'agence des acteurs dans un ordre institué (Breviglieri, Lafaye, \& Trom, 2009). En s'intéressant aux situations où les individus sont relativement libres dans leur capacité à exprimer leur point de vue, rappelons que l'approche de Boltanski et Thévenot laisse de côté les épreuves qui ne répondent pas à la possibilité de la justification. En situation de justification, l'épreuve met en scène les individus et leur capacité à qualifier les objets ou les personnes : il s'agit d'une épreuve de légitimité. Par exemple, un individu pourra contester le caractère légitime d'une filière de commerce équitable en regrettant son manque paradoxal d'esprit solidaire (monde civique). Ou, de la même façon, en demandant l'accroissement du caractère concurrentiel de cette même filière, on pourra supporter l'agencement social en place, ici par le recours à la Cité marchande. L'épreuve représente "le moment de mise en correspondance d'une action et d'une qualification, dans la visée d'une justification prétendant à validité générale » (Boltanski \& Thévenot, 1991, p. 410). La contestation d'un ordre se fait par l'intermédiaire des épreuves de légitimité tout comme sa cristallisation sous la forme du compromis : plus un état résiste aux épreuves, plus il apparaît stable. Plus récemment, Boltanski (2009, p. 158) a distingué parmi les épreuves légitimes plusieurs types d'épreuves parmi lesquels doivent être évoquées les épreuves de vérité et les épreuves de réalité. Les premières confirment la réalité en la déployant dans sa complétude. Dans le domaine de la notation du crédit par exemple, elles sont souvent caractérisées par le discours tautologique ou par l'utilisation de formules qui ne privilégient pas l'argumentation en se contentant d'énoncer ce qui est bon ou mauvais. Ainsi au début des années 2000, suite aux différentes crises impliquant les agences de notation, les acteurs de l'industrie répétaient que le système en place permettait la meilleure des régulations : une agence ne publierait jamais une note d'une entreprise si elle n'était pas dans le même temps persuadée de son objectivité (Taupin, 2012). Les épreuves de réalité en revanche confrontent ce qui est avec ce qui est prétendu être, elles supposent une argumentation approfondie. Dès 2008, suite à la crise des subprimes, la majorité des acteurs de l'industrie questionnent la réalité précédemment énoncée en doutant de l'oligopole en charge des notations (l'argumentation se fonde sur la Cité marchande) ou de l'exactitude de ces dernières (l'argumentation se fonde sur la Cité industrielle). Toutefois, les épreuves de réalité ne présument en rien de l'issue pour l'ordre régnant. Elles peuvent aussi bien renforcer qu'ébranler le compromis (Patriotta, Gond et Schultz, 2011 ; Taupin, 2012).
Luc Boltanski a récemment établi un nouvel agenda pour déployerla dimension critique delaSP(Boltanski, 2009; Boltanski, 2011). Il s'agit de développer une sociologie pragmatique de la critique en adoptant une position métacritique. La transposition d'un tel agenda au profit des CMS apparaît comme prometteuse (Dunne, 2012). Notre recherche propose ainsi de mobiliser la SP afin d'adopter une démarche critique au profit de la MOK. Il a toutefois été reproché à Boltanski l'aspect exclusivement théorique et général de son ambition (Fabiani, 2011, p. 405). A partir des outils tels que le compromis et l'épreuve, notre travail entend puiser dans les travaux de sciences de gestion existants s'inspirant de la SP pour contourner cette difficulté et fournir aux chercheurs de cette discipline une possibilité de mettre en œuvre la dimension critique de la SP.

\section{LES CMS EN SCIENCES DE GESTION}

Quelles sont les caractéristiques des études qui ont développé une perspective critique en sciences de gestion ? Fournier et Grey (2000) ont tenté de dégager des thèmes généraux autour de la notion de recherche « critique » en management. Premièrement les CMS partagent une visée de dénaturalisation. II s'agit de montrer que le statu quo n'est ni naturel, ni inévitable, et que les choses pourraient être autrement. Deuxièmement, la réflexivité de ces travaux concerne la posture du chercheur et sa relation à son objet d'étude en termes d'épistémologie, d'ontologie et de méthodologie. Les études critiques remettent en cause l'idée qu'une réalité externe au chercheur peut être saisie objectivement par un protocole de recherche ancré dans le positivisme, c'està-dire recherchant l'objectivité là où la construction de la MOK semble empreinte d'une subjectivité non avouée par le chercheur. Enfin, la démarche des CMS est non-performative : elle vise à s'extraire de la perspective privilégiant la rationalité instrumentale (d'un point de vue logique, chercher le rapport moyens/fin optimal) comme manière dominante de concevoir et de réaliser le fonctionnement des organisations. Afin de statuer sur le caractère " critique » d'une théorie, la théorie de l'ActeurRéseau (ANT) de Bruno Latour, Whittle et Spicer (2008) ont repris l'approche de Fournier et Grey pour en examiner les fondements ontologiques, épistémologiques et politiques.

Dans le domaine des sciences de gestion, l'opération critique est généralement réalisée par l'intermédiaire d'opérations de dévoilement (Golsorkhi, Leca, Lounsbury, \& Ramirez, 2009). Les CMS ont été inspirées par les axes théoriques des courants de la sociologie critique, de l'Ecole de Francfort, du marxisme et de ses variantes. Selon cette approche critique des MOK, la réalité serait dissimulée aux acteurs et le 
chercheur serait le seul à même de la révéler. On recherche alors la structure objective qui guide les acteurs dans la vie sociale. Dans ce cadre, les agissements des acteurs consistent en des réponses à l'existence de ces structures.

Cette conception des CMS a cependant été contestée comme laissant trop de place aux considérations épistémologiques (Thompson, 2004). Thompson et O'Doherty (2009) ont souligné les conséquences de cet état de fait : les CMS s'éloignent des pratiques et des acteurs organisationnels en ignorant largement les processus concrets de travail. De ce fait, la critique, perdant toute proximité avec la réalité vécue, ne parvient pas à remettre en cause les phénomènes de domination managériale (Thompson, 2004). Ces constatations ont mené à la redéfinition de la notion de performativité (Spicer, Alvesson, \& Kärreman, 2009 ; Alvesson \& Spicer, 2012) pour adopter la notion de "performativité critique ». Dans une perspective de performativité critique, le MOK doit chercher à s'extraire de l'analyse de l'aliénation dans la forme traditionnelle que nous venons de décrire. Alors que la performativité traditionnelle se contente de mettre en avant la manière par laquelle les discours sont mis en adéquation avec les structures de sens, la performativité critique insiste sur le processus selon lequel les discours sont « utilisés de manière active, parodiés et modifiés » (Spicer, Alvesson, \& Kärreman, 2009, p. 544, traduction de l'auteur). La performativité « implique une intervention active et subversive dans les discours et les pratiques managériaux » (Spicer, Alvesson, \& Kärreman 2009, p. 538, traduction de l'auteur). Alcadipani et Hassard (2010) ont retenu cette vision plus affirmative et positive de la critique pour déceler à leur tour le potentiel critique de la théorie de l'Acteur-Réseau en MOK. Spicer et autres (2009, p. 545) identifient cinq éléments constitutifs d'une approche de performativité critique : adopter une posture affirmative, une éthique du care, une orientation pragmatique, prendre en compte les potentialités et adopter une orientation normative. Nous les résumons dans le tableau 1 ci-contre.

Alvesson et Willmott (2012, p. 201, traduction de l'auteur) notent que la notion de performativité critique combine la théorie critique avec « une reconnaissance des contraintes du monde de l'entreprise et une compréhension plus empathique de la situation des managers et des autres acteurs des organisations, de leurs préoccupations et de leurs possibilités d'action ». Néanmoins, dans leur article séminal, si Spicer et autres exposent quelques pistes pour le développement d'une approche performative des CMS, ils insistent sur le caractère partiel et non définitif de leur illustration du concept, notamment lors de leur tentative de présentation des "tactiques" de la performativité critique (Spicer, Alvesson, \& Kärreman, 2009, p. 545).
TABLEAU 1. Les caractéristiques de la performativité critique selon Spicer et autres (2009)

\begin{tabular}{l|l}
\hline Caractéristique & Mise en œuvre \\
\hline Posture affirmative & $\begin{array}{l}\text { Positionnement à proximité de l'objet } \\
\text { de critique afin d'identifier les points de } \\
\text { révision potentiels. }\end{array}$ \\
\hline Ethique du care & $\begin{array}{l}\text { Permettre l'expression des opinions } \\
\text { des acteurs mais aussi chercher à les } \\
\text { questionner de manière subtile. }\end{array}$ \\
\hline Pragmatisme & $\begin{array}{l}\text { Travailler avec de aspects spécifiques } \\
\text { d'une organisation. }\end{array}$ \\
\hline Potentialités & $\begin{array}{l}\text { Créer un sens de ce qui pourrait être en } \\
\text { engageant des potentialités latentes } \\
\text { dans une organisation. }\end{array}$ \\
\hline Normativité & $\begin{array}{l}\text { Assertion systématique de critères } \\
\text { utilisés pour juger les bonnes formes } \\
\text { d'organisation. }\end{array}$ \\
\hline
\end{tabular}

Source : Spicer et autres (2009)

Plusieurs tentatives d'opérationnalisation de la notion de performativité critique ont été réalisées par les chercheurs en sciences de gestion. Dans leur approche critique du leadership, Alvesson et Spicer se sont focalisés sur la présentation de la situation réalisée par le manager lui-même et non sur les caractéristiques de ce dernier, telles que ses traits de caractère, ses valeurs ou ses compétences (Alvesson \& Spicer, 2012, p. 379). Dans un autre travail, Spicer et autres (2009, p. 549) proposent de mettre en œuvre l'éthique du care au travers d'une mystery-led approach. Pour le chercheur, il s'agit de travailler sur des mystères organisationnels en court-circuitant le traditionnel protocole de recherche fondé sur la théorie, afin de ménager un espace pour les conceptions des acteurs. Les chercheurs en CMS ont en effet tendance à chercher la confirmation de leurs propres théories au détriment de celles pouvant émerger de leur terrain empirique, en particulier lorsqu'elles infirment ces modèles théoriques bien établis. L'opérationnalisation de cette mistery led aproach demeure toutefois malaisée. Dans cet article nous arguons que, pour les études cherchant à s'inscrire dans la performativité critique décrite par Spicer et autres (2009), la démarche de la SP représente une théorie adéquate afin d'améliorer l'opérationnalisation de la compréhension empathique des acteurs. Car la SP développe une approche par laquelle le chercheur rend explicite les catégorisations des acteurs sans y voir a priori le résultat d'une illusion ou d'une contrainte. L'utilisation de la SP en CMS redonne même une place plus conforme que celle que le cadre de la performativité critique accorde à l'acteur. Les travaux étudiant la stupidité organisationnelle (Alvesson \& Spicer, 2012, Alvesson, \& Willmott, 
2012, p. 207), par exemple, ne nous semblent pas proposer une tactique permettant la mise en œuvre de l'alternative critique promise par l'approche de la performativité critique. Selon la théorie de la firme fondée sur la stupidité (Alvesson \& Spicer, 2012), le management met en veille ses capacités de réflexion critique. En comparaison, la SP apporte une forme de performativité critique plus positive puisqu'elle ne dénie pas les facultés critiques des individus dans son intervention subversive dans les discours managériaux. Dans les sections suivantes, nous nous chargerons donc de démontrer que l'étude du sens moral des acteurs de la SP permet de concrétiser la démarche de la performance critique. Nous nous inscrivons ainsi dans la lignée de travaux récents s'inspirant des nouvelles théories à disposition du chercheur critique en sciences de gestion (Huault, Perret, \& Spicer, 2014) afin de ménager une voie de sortie dans le dilemme opposant, au sein de toute démarche critique, l'orientation radicale et l'orientation pragmatique.

\section{LA DIMENSION CRITIQUE DE LA SP}

Afin de faire ressortir la dimension critique de la SP, nous discutons de l'articulation des concepts de la SP utilisés dans les recherches en gestion avec les caractéristiques de la performativité critique précédemment énoncées.

Avant toute chose, la SP propose de s’intéresser à ce que les acteurs disent en étudiant les mondes constitués, c'est-à-dire l'appréhension des objets et des personnes selon un ou plusieurs principes issus des cités. Pour les MOK, la question posée est la suivante : selon quels termes les acteurs appréhendent-ils leur réalité organisationnelle ? Il s'agit, d'un point de vue méthodologique, de donner la primauté aux catégorisations des acteurs, pour, dans un second temps parvenir aux interprétations du chercheur. La SP fournit ainsi une mise en œuvre des notions de posture affirmative et de l'éthique du care.

La réflexion épistémologique, qui fait de la SP « une science de la science implicite des acteurs » (Karsenti, 2012, p. 570), mène cette approche au plus près de leurs pratiques afin d'y identifier des potentiels d'émancipation (posture affirmative). Dans ce cadre le chercheur tente de mettre en lumière la manière dont les acteurs interprètent leurs faits et gestes à travers leurs propres catégorisations. La « catégorisation » englobe le processus de mise en relation des actions avec les catégories/ structures plus générales (voir la figure 2). La SP permet alors de concevoir des protocoles de recherche visant à se rapprocher de l'objet analysé. En recourant à la SP, Reinecke rend explicite les principes qui ont guidé la fixation des prix dans le domaine du Commerce Equitable (Reinecke, 2010). Elle donne à voir les catégorisations établies par les acteurs eux-mêmes dans ce processus (éthique du care). La cité industrielle et la cité civique ont été mobilisées afin de définir un principe supérieur justifiant l'établissement de calcul (calculation). Chez Reinecke, l'étude de la recherche d'un compromis autour de l'établissement du prix des produits permet de cibler avec plus de précision les acteurs centraux dans le phénomène de domination. La recherche révise par exemple le rôle traditionnellement associé aux figures dominantes de l'industrie dans la mobilisation des principes supérieurs : «Je m'attendais à ce que les représentants des négociants soient ceux qui combattraient le plus âprement la hausse des prix. Mais ce ne fut pas le cas » (Reinecke, 2010, p. 572, traduction de l'auteur).

Le caractère pragmatique, inclus dans l'énonciation même de la SP, indique que cette approche met l'action au centre de l'analyse en se focalisant sur ce que l'homme fait de lui-même et des autres (Bénatouïl, 1999). Selon le point de vue pragmatique développé par la performativité critique, il faut entreprendre des observations adaptées au contexte et à ses contraintes propres. Dans la SP, la cité idéelle est ignorée au profit de l'agencement composite en place. Du point de vue des CMS, l'agencement en place s'impose aux acteurs : dans une certaine mesure, la cité industrielle (la performance) s'impose aux organisations. Cependant, dans le cas de l'incident nucléaire étudié par Patriotta et autres (2011), la controverse autour de l'évaluation des risques de l'accident s'étend à de nombreux acteurs ayant un intérêt au débat. Elle évolue au-delà d'une référence initiale à la cité industrielle. La controverse se déplace vers les cités domestiques, civiques et marchandes. La SP a permis de décrire le processus où se sont succédé les configurations d'ordre social pour préserver la légitimité des institutions existantes suite à l'accident nucléaire. Ainsi la perpétuation du statu quo dans l'industrie nucléaire s'est faite ici au prix d'un réajustement du compromis en vigueur. En s'intéressant aux sphères d'exercice de la justice, la SP se focalise sur les espaces où les participants ayant un intérêt s'impliquent dans le débat, conformément à l'orientation pragmatique de performativité critique. Dans le cas de Patriotta et autres, le fait de donner voix, dans un deuxième temps, au monde civique, au monde domestique et, enfin, au monde vert, montre un processus de domination plus complexe que la simple présence d'un système tout-puissant favorisant certaines entités.

La SP s'accorde avec la notion de potentialité. Selon la performativité critique, l'idée de potentialité telle qu'exprimée par Chua (1986, p. 619, traduction de l'auteur) reconnait que les « êtres humains ne sont pas réduits à exister dans un état particulier ; leur être et leur environnement matériel ne sont 
pas épuisés par leur circonstance immédiate ». Les épreuves de la SP sont des sources d'émancipation de ce type : elles sont susceptibles de réduire les contraintes qui s'exercent sur les individus dans les organisations. Le sens du social des acteurs organisationnels les mène à faire appel à la dimension civique et industrielle dans un univers marchand (Reinecke, 2010) ou à mobiliser la dimension verte ou civique dans un univers industriel (Patriotta, Gond, \& Schultz, 2011). Autrement dit, dans le cadre de la SP, les acteurs sont compétents : ils sont capables de faire valoir leur conception du juste relativement indépendamment des contraintes.

Wissler (1989) mobilise la SP pour l'étude des établissements de crédit implantés dans des zones rurales. Cette analyse donne un exemple de la manière dont la SP extrait les acteurs de leur situation immédiate pour s'engager dans des potentialités. Là où nous pourrions penser la gestion des banques comme relevant de l'unique logique industrielle ou marchande, il apparaît que l'ancrage dans le tissu local s'intègre dans le fonctionnement de ces agences. Dans les zones rurales, des compromis sont mis en place (Wissler, 1989) pour permettre des points de passages entres les logiques relevant du monde domestique (l'ancrage local incarné par les relations amicales, familiales, de voisinage, de services) et celles relevant du monde industriel (volonté d'objectiver la décision d'octroi de crédit). L'étude du processus d'octroi de crédit montre comment l'établissement bancaire a dû faire un compromis entre ces deux logiques d'action dans son fonctionnement (Wissler, 1989, p. 113). A travers ses recommandations, le conseil d'administration local s'engage dans une logique de jugement domestique sans se limiter à la simple réalisation de l'analyse financière, relevant du monde industriel. Dans son interprétation du social, la SP nourrit les potentialités, Juhem (1994, p. 86) note que, même dans les usines qui sont organisées selon les principes de la cité industrielle, " les autres principes de justification ne deviennent pas caducs dans une situation dominée par le mode d'organisation d'une seule cité ". Les acteurs sont toujours susceptibles de saisir les objets non pertinents dans le monde qui les entoure. Comme l'indiquent Boltanski et Thévenot (1991, p. 268), un employé qui se fait licencier dans le bureau de son patron, un univers industriel, peut se saisir de la photographie de la famille de son supérieur trônant sur le bureau : « elle peut aussi être relevée de façon à faire surgir un autre monde et un principe de justice domestique dont la prise en compte pourrait atténuer la rigueur du verdict : “Moi aussi, comme vous, j’ai des enfants"».

En se focalisant sur les critères de l'action juste, la SP permet de reformuler la discussion autour de l'éthique, en particulier du bien. Sans pour autant proposer des valeurs qui valent en toute place et en toute époque, la SP reconnait l'existence de conflits dans la vie organisationnelle et rend clair les critères retenus pour déterminer qu'une situation soit juste. Dans l'acception la plus pauvre du terme, la SP n'a pas d'ambition normative : « elle ne prétend pas que les topiques dont elle repère les effets et les origines dans un débat soient ses cadres normaux ou idéaux, et c'est paradoxalement pour cette raison qu'elle peut intervenir dans le débat » (Bénatouïl, 1999, p. 303). La démarche de la SP n'est pas normative dans un sens réduit du terme où elle annoncerait le bien dans un absolu. En revanche, à travers sa démarche systématique d'analyse des répertoires (Silber, 2003), la SP permet la création des conditions de l'émergence du bien : « L'intervention pragmatique aide les participants de droit au débat, en particulier ceux qu'on y entend de fait le moins, à entrer en possession de leur propre position politique, c'est-à-dire à en mobiliser et en exprimer la spécificité » (Bénatouïl, 1999, p. 304). La SP intervient pour cibler puis combler l'écart qui existe lorsque les dispositifs ne permettent pas la réalisation du sens moral des acteurs. Le travail de Taupin (2012) présente une illustration du caractère prometteur de cette posture pour les CMS. En mobilisant le cadre de la SP, le chercheur étudie l'industrie de la notation du crédit lors de la crise des subprimes. Alors que les acteurs qui s'impliquent dans la réforme réglementaire de la notation du crédit cherchent à favoriser la compétition, à atteindre la performance et à améliorer la transparence, Taupin montre le processus qui mène à ce que l'industrie de la notation demeure une industrie oligopolistique où les trois principales agences réalisent $98 \%$ du chiffre d'affaire mondial de l'activité, où les notations se révèlent très souvent inexactes et enfin, où l'opacité du véritable fonctionnement de la notation reste profonde.

\section{UNE NOUVELLE ONTOLOGIE CRITIQUE AU PROFIT DE LA MOK}

Nous pouvons à ce stade rapprocher la SP des caractéristiques de la CMS énoncées par Fournier et Grey (2000). Il est aisé de distinguer une ontologie de dénaturalisation dans la SP. Au travers de la description du social qu'elle propose, à partir de la critique de la réalité élaborée par les acteurs, cette sociologie donne à voir que les choses pourraient être autrement. Premièrement, le compromis et le format d'épreuve qui sous-tendent cette réalité sont le résultat d'un accord qui s'est construit d'une manière spécifique et, surtout, dans un rapport à un monde qui est ontologiquement considéré comme insaisissable et instable. Selon Boltanski, le compromis, par nature composite, est intrinsèquement fragile. Comme nous l'avons vu, dans les zones rurales françaises, la manière dont l'établissement de crédit résout 
la contradiction entre les logiques marchande et domestique tient à la réalisation d'un compromis fragile, contestable et spécifique. Dans un second temps, en insistant sur la liberté des acteurs, cette théorie s’intéresse à leur capacité à s'extraire des situations. Les principes de justification ne deviennent pas caducs dans un dispositif donné car les acteurs sont doués de compétence critique. Les dispositifs sont donc variés car ils correspondent au résultat de ce travail créatif de critique. Ainsi, en prenant pour objet ces dispositifs dans leur grande variété, la SP accomplit un véritable travail de dénaturalisation.

Pour autant, la SP ne conçoit pas cette dénaturalisation comme une « révélation » du chercheur, conformément aux caractéristiques de son cadre épistémologique. En effet, initialement, la SP s'est précisément développée autour d'une épistémologie augmentant le degré d'exigence habituel des épistémologies critiques. Lorsqu'il aborde les qualifications des acteurs, le chercheur en CMS qui mobilise la SP ne s'interroge pas sur le statut de vérité des affirmations, en cherchant des critères qui lui permettraient de mettre en doute ou de valider les représentations qui lui sont transmises (Boltanski, 2012, p. 341). De cette manière, il s'agit également d'éviter de conférer un rôle trop surplombant au chercheur. Ce dernier est placé au même niveau que celui des acteurs qu'il étudie, il ne dévoile pas un illusio (Golsorkhi \& Huault, 2006 ; Golsorkhi, Leca, Lounsbury, \& Ramirez, 2009) dont lui-seul peut constater la présence. La SP s'inscrit dans une épistémologie réflexive différente par exemple de celle de la sociologie critique mais qui partage néanmoins avec elle le refus du positivisme méthodologique (Bénatouïl, 1999). Chez Boltanski la réalité n'existe pas de manière extérieure (Boltanski, 2009), les compromis et les formats d'épreuves ne sont qu'un moyen de stabiliser le réel par nature irréductible afin de permettre aux acteurs d'agir. La SP répond donc directement au souci exprimé en management de ne pas placer la morale du chercheur au-dessus de celle des acteurs (Czarniawska, 2005). En ce sens, la SP en CMS accorde selon nous une place plus conforme à la manière dont l'acteur est théorisé dans la notion de performativité critique, notamment en regard de tentatives récentes d'illustration empirique de cette notion. Dans l'article d'Alvesson et Spicer (2012) par exemple, on considère que le management met en veille ses capacités de réflexion critique. Au contraire de cette recherche, la SP apporte une forme de performativité critique plus positive (Alcadipani \& Hassard, 2010) puisqu'elle ne dénie pas les facultés critiques propres aux individus dans son intervention subversive dans les discours managériaux.

La SP peut enfin être qualifiée de non performative et non d'anti-performative. Tout en proposant plusieurs sources de justifications alternatives, la SP ne s'oppose pas par principe à la réflexion instrumentale : elle n'est pas anti-performative. Elle fait une place à la mobilisation, par les acteurs, de la science pour améliorer la performance d'un phénomène. Elle ne nie pas le recours nécessaire à ce type de raisonnement d'optimisation des moyens en fonction des fins par le sens moral des acteurs. L'entreprise est reconnue comme un compromis industrielmarchand (Boltanski \& Thévenot, 1991) puisqu'il faut bien un calcul moyen/fins pour permettre aux passagers des compagnies aériennes d'arriver à l'heure (Spicer, Alvesson, \& Kärreman, 2009, p. 543).

Dans la section précédente, nous avons indiqué que la SP peut être rapprochée des caractéristiques de la performativité critique. Parmi celles-ci, l'aspect normatif de la démarche de SP interroge le plus. A cet égard, la SP ouvre une nouvelle voie au profit des CMS. Le critère systématique utilisé pour juger une bonne forme d'organisation réside dans l'analyse de l'adéquation entre, d'une part, ce que la réalité organisationnelle est, et, d'autre part, ce que sont les attentes morales des individus composant ces organisations. Avec la SP de la critique en effet, on prend « appui sur [le sens moral des acteurs] et, particulièrement, sur leur sens ordinaire de la justice, pour rendre manifeste le décalage entre le monde social tel qu'il est et ce qu'il devrait être pour satisfaire aux attentes morales des personnes »(Boltanski, 2009, p. 56 ; Boltanski, 2011). Il s'agit ici d'une petite rupture par rapport au programme initial de la SP, puisque « le sociologue ne se contente plus de 'suivre les acteurs' dans la désingularisation de leurs plaintes et le 'montage' de leurs affaires » (Auray, 2012, p. 2). Tout en émettant une critique, les recherches de SP des CMS se placent au niveau des acteurs pour saisir leurs représentations du monde organisationnel. En cela l'on peut rapprocher cette démarche de la performativité critique et de son souci de se placer au plus près de l'objet de la critique. Parvenir à extraire la dimension critique de la SP suppose toutefois la mise en œuvre d'un double mouvement de rapprochement de l'objet puis d'une forme de distanciation critique. Comme pour la performativité critique, il ne suffit pas d'adopter toutes les règles associées avec l'objet de la critique (Spicer, Alvesson, \& Kärreman, 2009, p. 546). La réalisation d'un second mouvement, impliquant plus spécifiquement les caractéristiques pragmatique et normative de la performativité critique, est nécessaire ; il rend l'opération rigoureuse.

L'apport de nouvelles théories issues des sciences humaines et sociales pour la communauté des chercheurs en CMS a déjà été démontré, notamment pour ce qui concerne l'ANT (Alcadipani \& Hassard, 2010). Dans leur opération critique la SP et l'ANT présentent des points de convergence :

Au plus loin des sociologies critiques, il ne s'agit pas de dévoiler ce que nous ne voyons pas (et 
que seul le sociologue aurait le mérite de venir nous dévoiler), mais de dévoiler ce que nous voyons déjà sans nous le dire, et surtout sans le dire aux autres, à même la façon dont nous agissons et pensons. (Karsenti, 2012, p. 570).

Néanmoins les deux approches divergent dans leur démarche de recherche (Latour, 2009, Karsenti, 2012). Une critique faite à l'ANT réside dans la tendance à privilégier une approche critique par laquelle le chercheur réalise une généalogie de l'instanciation de la puissance légiférante. Or comme l'indique Karsenti (pp. 570-571) « la critique peut se conduire en creusant une forme d'écart qui ne soit pas le levier d'une juridiction ou d'une démystification de surplomb (c'est tout l'effort de la [SP] que de l'aménager) ». Selon Karsenti la critique émise par l'ANT est celle d'un serviteur des serviteurs et non celle d'un serviteur du roi : le chercheur se positionne au plus près des acteurs d'une manière qui limite l'ampleur de la critique exercée en privilégiant une critique au sens juridictionnel. L'opération critique de l'ANT a également été stigmatisée pour sa difficile opérationnalisation (Karsenti, 2012, p. 571). En se reposant sur les concepts aujourd'hui stabilisés de compromis et d'épreuve, la SP évite cet écueil en mettant à disposition du chercheur en CMS un outillage validé empiriquement et aisément opérationnalisable.

\section{DOMINATION SIMPLE ET DOMINATION GESTIONNAIRE COMPLEXE}

Nous avons constaté qu'il était possible d'adopter une démarche de CMS en mobilisant la SP. Lorsqu'elle est pleinement exploitée, la SP non seulement dénaturalise de manière réflexive, elle opère en outre une rupture avec les formes précédentes de critique en management. Les CMS se sont focalisées sur l'analyse des formes traditionnelles de domination par lesquelles les acteurs, dotés de faibles capacités critiques, sont dominés sans le savoir. Dans ce cadre, la quasi-totalité des conduites des acteurs sont expliquées par l'intériorisation de normes dominantes surtout au cours du processus d'éducation. Ainsi les CMS ont-elles expliqué la domination masculine au travail (Martin, 1990), l'utilisation du langage comme facteur de reproduction du pouvoir (Alvesson \& Willmott, 2003), la perpétuation de la domination occidentale à travers l'impérialisme de la diffusion du management (Frenkel \& Shenhav, 2003). De ce fait les situations dans lesquelles les acteurs se trouvent plongés sont négligées (Boltanski, 2009, p. 42). Ainsi l'adoption de la responsabilité sociale des entreprises par les managers dépendrait des prédispositions sociales de ces derniers et des différentes formes de capital dont ils disposent (Aaken, Splitter, \& Seidl, 2013). Dans leur opération critique de dévoilement, ces études relevant des CMS accordent une position surplombante au chercheur et cela entre en conflit avec leur objectif d'émancipation (Huault, Perret, \& Spicer, 2013). Les sociologies critiques, dont s'inspirent les CMS, ne parviennent pas à prendre l'homme de la pratique pour ce qu'il est, c'est-à-dire à le considérer comme pensant et agissant à l'état pratique (Karsenti, 2011). Or les mécanismes d'oppression ont évolué et leur analyse nécessite de dépasser les approches qui se focalisaient sur ces phénomènes de domination simples. De ce fait, les CMS ne réussissent pas à saisir les conditions contemporaines de domination complexe fondées sur la pratique, comme, par exemple lorsque la classe dominante est la classe qui se place dans une position, inaccessible à la majorité, de double relation vis-à-vis des règles qui s'imposent (Dunne, 2012). L'analyse articulant SP et approche performative de la critique accorde une place centrale, et non périphérique aux compétences des acteurs et aux dispositifs dans lesquels leurs actions prennent place, contrairement à l'approche traditionnelle focalisée sur les structures. Le cadre de la SP permet de mettre à jour une forme de domination qui repose sur une augmentation de la complexité des formes de coordination (Fabiani, 2011) par l'étude du processus de catégorisation au cours duquel les équivalences sont opérées par les acteurs. La catégorisation « soutient la nouvelle capacité générale et forme une possible source de pouvoir abusif » (Blokker \& Brighenti, 2011, p. 395). Dans ce cadre, ce n'est pas une forme de violence qui impose la fin du débat mais un processus de justification. La résolution d'une controverse dépend des capacités des uns et des autres à imposer un ou plusieurs principes supérieurs ; «les personnes reconnaissent certaines inégalités comme légitimes et en dénoncent d'autres comme injustes dans certaines situations et au nom de certains principes : c'est à travers ces actions de reconnaissance et de dénonciation que l'approche pragmatique peut fournir des modèles au sens commun de la domination » (Bénatouïl, 1999, p. 307).

Le domaine du management et des études organisationnelles s'est inspiré des prolongements de la SP développés dans le Nouvel Esprit du Capitalisme (Boltanski \& Chapiello, 1999). Cet ouvrage est devenu central pour l'étude des changements contemporains dans la vie économique et organisationnelle (Du Gay \& Morgan, 2013) notamment en tant qu'il permettait l'analyse des formes du néo-libéralisme. Cependant l'analyse de Boltanski et Chiapello correspond aux expressions du capitalisme observées entre 1970 et 1990. Il ne donne pas de clés concrètes pour entreprendre une analyse 
de sociologie pragmatique critique des nouvelles formes du capitalisme financier et des nouvelles formes de domination complexe qu’il génère (Willmott, 2013). De plus, en se focalisant sur l'inclusion de la critique dans la domination, l'approche issue du Nouvel Esprit du Capitalisme empêche toute véritable forme de critique (Martin, 2013). L'outillage conceptuel que nous avons présenté permet une problématisation politique plus nette des caractéristiques des dispositifs gestionnaires (Friedlender, 2010, p. 16 ; Dunne, 2012). En effet, les sociétés modernes sont marquées par une domination complexe que Boltanski qualifie de gestionnaire.

Alors que les régimes de domination simples, fonctionnant sur l'oppression, reposent sur le fait que leurs institutions cérémonielles cherchent à écarter la réalité, les sociétés gestionnaires placent au centre les processus de modification de la réalité, et par l'identification de ce nouveau format de réalité à quelque chose de nécessaire. (Auray, 2012, p. 5).

Boltanski (2012) fournit par exemple aux CMS un outil privilégié pour décrire le «triomphe » de la figure de l'expert. Au centre du dispositif de domination complexe, l'expert disqualifie les représentations alternatives et légitime la réalité qu'il édicte (Auray, p. 5). Ainsi, avec les classements, nous assistons à une transformation des contours de la réalité sous forme d'une orthodoxie de la norme : le format d'épreuve établit un mode de gouvernance instrumental gestionnaire qui favorise les experts (Friedlender, 2010, p. 16). L'établissement de formats d'épreuve mouvants complique la tâche de la critique de contester leur validité. Les dispositifs n'ont plus vocation à maintenir une réalité fondée sur des qualifications correspondant à des formats d'épreuves établis. Le déplacement constant d'un format à un autre représente le mode de fonctionnement. L'écart entre la réalité et le monde devient alors difficilement repérable de l'extérieur. Boltanski évoque l'exemple du benchmarking « la fabrication et la publication de palmarès permettant de hiérarchiser des organisations (...) en fonction d'une norme d'efficacité » (2009, p. 199) en tant que processus complexe facteur de domination (un effet de bouclage réflexif). Il expose la manière dont ces dispositifs, qualifications et format d'épreuves reconnus et établis, transforment la réalité en un mode de gouvernance instrumental gestionnaire. Et, dans ce dispositif, certains acteurs organisationnels plus avisés et mieux dotés comme les consultants, les hauts fonctionnaires et certains acteurs de terrain prennent une place centrale. Autrement dit, les conditions établies pour l'exercice du sens de la justice des acteurs font que ce sont toujours les mêmes qui, face aux épreuves, se révèlent médiocre ou au contraire satisfont à la plupart des épreuves (Boltanski, 2009, p. 67 ; Boltanski, 2011).

\section{CONCLUSION}

Dans cet article, nous avons cherché à montrer que la SP proposait une ontologie, une épistémologie et un rapport à la performativité permettant une approche critique au profit de la MOK. Il est apparu que les limites des approches développant une théorie critique des organisations pouvaient également être surmontées par le recours à la SP. En mobilisant cette théorie, les difficultés rencontrées par les approches critiques en management (position surplombante du chercheur, éloignement de l'objet étudié, faible intérêt accordé aux représentations des acteurs etc...) sont dépassées au profit de la réalisation d'une critique correspondant à la notion de critique performative (Spicer, Alvesson, \& Kärreman, 2009). L'utilisation de la SP met à disposition des outils concrets pour le chercheur critique en sciences de gestion. L'utilisation des notions de compromis et d'épreuve dans une démarche générale en deux temps, faisant d'abord l'hypothèse de relative liberté des acteurs dans un premier temps - pour ensuite expliciter le processus de domination s'exerçant - dans un deuxième temps - permet la mise en avant de processus de domination complexe. Ainsi parvient-on à analyser la manière dont les acteurs convergent dans leurs actions, établissant des conditions de domination, indépendamment des positions spatiales ou institutionnelles qu'ils occupent ou des inégalités de pouvoir en termes de propriété et de capital.

La SP ouvre la voie à de futures recherches dans le domaine des CMS que nous résumons à deux axes. Cette approche mène premièrement à distinguer les situations de domination simples, dans lesquelles s'applique un pouvoir arbitraire, des situations de domination complexe. Selon Boltanski, le monde du management est caractéristique de cette domination complexe où le changement est la règle, où l'on a foi dans la capacité humaine à avoir une prise sur le monde. La domination complexe met en scène des acteurs à la poursuite constante de nouvelles réalités (Dunne, 2012). Si Boltanski (2009, 2011) développe quelques pistes théoriques, les études détaillées portant sur ces phénomènes de domination sur le lieu de travail restent à écrire. Deuxièmement, la SP fournit un cadre permettant de reconsidérer la manière dont les CMS conçoivent l'émancipation. En reconnaissant la réflexivité des acteurs, leur compétence au jugement, il ne s'agit pas de révéler aux individus les réelles causes de leur domination mais de considérer les acteurs capables de créer comme de rompre les conditions de leur domination. Pour le chercheur, il s'agit d'étudier tous les individus et le groupes qui s'engagent dans un travail de justification pour analyser les critiques du management, positives ou négatives. Les sources peuvent prendre la forme 
de lettres et de courriels envoyés aux entreprises et aux journaux (comme Boltanski l'a lui-même fait), afin d'étudier la constitution de catégories institutionnalisées dans lesquelles les individus se reconnaissent. Cela peut également conduire à prendre en compte les nombreux forums de consultation mis à disposition des acteurs avant l'élaboration d'une régulation. Une telle étude ne se contenterait pas d'étudier le résultat de la consultation en termes d'actions menées : il s'agirait de considérer si la manière dont les problèmes sont appréhendés est conforme au sens que les acteurs ont eux-mêmes exprimé.

\section{RÉFÉRENCES}

Aaken, D., Splitter, V., \& Seidl, D. (2013). Why do corporate actors engage in pro-social behavior? A Bourdieusian perspective on corporate social responsibility. Organization, 20(3), 349-371.

Alcadipani, R. \& Hassard, J. (2010). Actor-network theory, organizations and critique: towards a politics of organizing. Organization, 17(4), 419-435.

Alvesson, M. \& Spicer, A. (2012). Critical leadership studies: the case for critical performativity. Human Relations, 65(3), 367-390.

Alvesson, M. \& Spicer, A. (2012). A stupidity-based theory of organizations. Journal of management studies, 49(7), 1194-1220.

Alvesson, M. \& Willmott, H. (2003). Studying management critically: SAGE Publications Limited.

Alvesson, M. \& Willmott, H. (2012). Making sense of management: a critical introduction. Second edition. London: Sage.

Annisette, M. \& Richardson, A. J. (2011). Justification and accounting: applying sociology of worth to accounting research. Accounting, Auditing \& Accountability Journal, 24(2), 229-249.

Auray, N. (2012). La sociologie en rouge et noir. La Vie des Idées, novembre.

Bénatouïl, T. (1999). Critique et pragmatique en sociologie : quelques principes de lecture. Annales. Histoire, sciences sociales, 54(2), 281317.

Bénatouïl, T. (1999). A tale of two sociologies: the critical and pragmatic stance in contemporary French sociology. European Journal of Social Theory, 2(3), 379-396.

Biggart, N. \& Beamish, T. (2003). The economic sociology of conventions: habit, custom, practice, and routine in market order. Annual Review of Sociology, 29, 443-464.

Blokker, P. (2011). Pragmatic sociology: theoretical evolvement and empirical application. European Journal of Social Theory, 14(3), 251-261.

Blokker, P. \& Brighenti, A. (2011). An interview with Laurent Thévenot: on engagement, critique, commonality, and power. European Journal of Social Theory, 14(3), 383-400.

Boivin, C. \& Roch, J. (2006). Dominant organizational logic as an impediment to collaboration. Management Decision, 44(3), 409422.

Boltanski, L. (2009). De la critique : Précis de sociologie de l'émancipation. Paris: Gallimard.
Boltanski, L. (2011). On critique: a sociology of emancipation. Cambridge: Polity Press.

Boltanski, L. (2012). Enigmes et complots : une enquête à propos d'enquêtes. Paris: Gallimard.

Boltanski, L. \& Chiapello, E. (1999). Le nouvel esprit du capitalisme. Paris: Gallimard.

Boltanski, L. \& Thévenot, L. (1991). De la justification. Les économies de la grandeur. Paris: Gallimard.

Breviglieri, M., Lafaye, C., \& Trom, D. (2009). Compétences critiques et sens de la justice, Colloque de Cerisy. Paris: Economica, Collection Etudes Sociologiques.

Breviglieri, M., Lafaye, C., \& Trom, D. (2009). Sociologie pragmatique et normativité de l'agir en public. In Breviglieri M., Lafaye C., \& Trom D. (Eds) Compétences critiques et sens de la justice, Colloque de Cerisy. Paris: Economica, Collection Etudes Sociologiques.

Chua, W. F. (1986). Radical developments in accounting thought. The Accounting Review, 61(4), 601-632.

Cloutier, C. \& Langley, A. (2013). The logic of institutional logics: insights from French pragmatist sociology. Journal of Management Inquiry, 22(4), 360-380.

Czarniawska, B. (2005). En teori om organisering. Lund: Studentlitteratur.

Dansou, K. \& Langley, A. (2012). Institutional work and the notion of test.M@n@gement,15(5),503-527.

Denis, J. L., Langley, A., \& Rouleau, L. (2007). Strategizing in pluralistic contexts: rethinking theoretical frames. Human Relations, 60(1), 179-215.

Du Gay, P. \& Morgan, G. (2013). Understanding capitalism: crises, legitimacy and change through the prism of the new spirit of capitalism. In du Gay P. \& Morgan G. (Eds), New Spirits of Capitalism? Crises, Justifications, and Dynamics. Oxford: Oxford University Press.

Dunne, S. (2012). Stephen Dunne reviews on critique: a sociology of emancipation by Luc Boltanski. Organization, 19(4), 525-527.

Fabiani, J. L. (2011). Luc Boltanski, De la critique : précis de sociologie de l'émancipation. European Journal of Social Theory, 14(3), 401-406.

Fournier, V. \& Grey, C. (2000). At the critical moment: conditions and prospects for critical management studies. Human relations, 53(1), 7-32.

Frenkel, M. \& Shenhav, Y. (2003). From Americanization to colonization: the diffusion of productivity models revisited. Organization Studies, 24(9), 1537-1561.

Friedlender, J. M. (2010). La note critique sur De la critique, précis de sociologie de l'émancipation. Bulletin Sociologie de la Gestion, $30(5)$.

Fronda, Y. \& Moriceau, J. L. (2008). I am not your hero: change management and culture shocks in a public sector corporation. Journal of Organizational Change Management, 21(5), 589-609.

Golsorkhi, D. \& Huault, I. (2006). Pierre Bourdieu : critique et réflexivité comme attitude analytique. Revue française de gestion, 6(165), 15-34.

Golsorkhi, D., Leca, B., Lounsbury, M., \& Ramirez, C. (2009). Analyzing, accounting for and unmasking domination: on our role as scholars of practice, practitioners of social science and public intellectuals. Organization, 16(6), 779-797.

Huault, I., Perret, V., \& Spicer, A. (2014). Beyond macro- and microemancipation: rethinking emancipation in organization studies. Organization, 21(1), 22-49. 
Huault, I. \& Rainelli-Weiss, H. (2011). A Market for weather risk? Conflicting metrics, attempts at compromise and limits to commensuration. Organization Studies, 32(10), 1395-1419.

Jagd, S. (2011). Pragmatic sociology and competing orders of worth in organizations. European Journal of Social Theory, 14(3), 343-359.

Juhem, P. (1994). Un nouveau paradigme sociologique ? A propos du modèle des " économies de la grandeur " de Luc Boltanski et Laurent Thévenot. Scalpels, Cahiers de Sociologie Politique de Nanterre, 1, 115-142.

Karsenti, B. (2011). De Marx à Bourdieu. Les dilemmes du structuralisme de la pratique. In M. d. Fornel. \& A. Ogien (Ed.), Raison pratiques : Bourdieu. Théoricien de la pratique. Les éditions de l'EHESS, 103-134.

Karsenti, B. (2012). «Tenir au monde, le faire tenir » : linéaments pour une philosophie de l'attention. Archives de Philosophie, 4(75), 567-586.

Latour, B. (2009). Dialogue sur deux systèmes de sociologie. Colloque de Cerisy. In Breviglieri M., Lafaye C., \& Trom D. (Eds), Compétences critiques et sens de la justice, Colloque de Cerisy. Paris: Economica, Collection Etudes Sociologiques.

Martin, J. (1990). Deconstructing organizational taboos: the suppression of gender conflict in organizations. Organization Science, 1(4), 339-359.

McInerney, P. B. (2008). Showdown at Kykuit: Field-configuring events as loci for conventionalizing accounts. Journal of Management Studies, 45(6), 1089-1116.

Nachi, M. (2006). Introduction à la sociologie pragmatique. Paris: Armand Colin.

Patriotta, G., Gond, J. P., \& Schultz, F. (2011). Maintaining legitimacy: controversies, orders of worth and public justifications. Journal of Management Studies, 48(8), 1804-1836.

Pernkopf-Konhaeuser, K. \& Brandl, J. (2010). How should human resources be managed? From comparing models of staff development in a German and Russian professional service firm: a conventionalist approach. European Journal of Cross-Cultural Competence and Management, 1(4), 356-377.

Reinecke, J. (2010). Beyond a subjective theory of value and towards a "fair price": an organizational perspective on Fairtrade minimum price setting. Organization, 17(5), 563-581.

Ricoeur, P. (1991). Pour une éthique du compromis (entretien avec Paul Ricoeur). Alternatives non violentes, 80, 2-7.
Roch, J. M. (2005). A new way to look at the integration challenge: the reconciliation of collective representations. Advances in Mergers \& Acquisitions, 4, 23-50.

Silber, F. I. (2003). Pragmatic sociology as cultural sociology: beyond repertoire theory? European Journal of Social Theory, 6(4), 427-449.

Spicer, A., Alvesson, M., \& Kärreman, D. (2009). Critical performativity: the unfinished business of critical management studies. Human relations, $62(4)$, 537-560.

Stark, D. (2000). For a sociology of worth. Keynote address for the Meetings of the European Association of Evolutionary Political Economy, Berlin.

Stark, D. (2009). The sense of dissonance: accounts of worth in economic life. Princeton: Princeton University Press.

Taupin, B. (2012). The more things change... Institutional maintenance as justification work in the credit rating industry. M@n@gement, 15(5), 529-562.

Thévenot, L., Moody, M., \& Lafaye, C. (2000). Forms of valuing nature: arguments and modes of justification in French and American environmental disputes. In Lamont M. \& Thévenot L. (Eds), Rethinking comparative cultural sociology: Repertoires of Evaluation in France and the United States. Cambridge, MA: Cambridge University Press.

Thompson, P. (2004). Brands, boundaries and bandwagons: a critical reflection on critical management studies. In Fleetwood S. \& Ackroyd S. (Eds), Critical realist applications in organization and management studies. London: Routledge.

Thompson, P. \& O'doherty, D. (2009). Perspectives on labor process. In Alvesson M., Bridgman T., \& Willmott H. (Eds), Handbook of critical management studies. London: Sage.

Thornton, P. H. \& Ocasio, W. (2008). Institutional Logics. In Greenwood R., Oliver C., Sahlin K., \& Suddaby R. (Eds), The Sage Handbook of Organizational Institutionalism: Sage Publications.

Whittle, A. \& Spicer, A. (2008). Is actor network theory critical? Organizaiton studies, 29(1), 611-629.

Wissler, A. (1989). Les jugements dans l'octroi de crédit. In Boltanski L. \& Thévenot L. (Eds), Justesse et justice dans le travail. Paris: Cahiers du C.E.E. PUF.

Willmott H. (2013). Spirited away: when political economy becomes culturalized. In du Gay P. \& Morgan G. (Eds), New Spirits of Capitalism? Crises, Justifications, and Dynamics. Oxford: Oxford University Press. 